Published in "Physica A: Statistical Mechanics and its Applications 450: 486-496, 2016"

which should be cited to refer to this work.

\title{
Study of market model describing the contrary behaviors of informed and uninformed agents: Being minority and being majority
}

\author{
Yu-Xia Zhang ${ }^{\mathrm{a}, \mathrm{b}}$, Hao Liao ${ }^{\mathrm{b}, \mathrm{e}, *}$, Matus Medo $^{\mathrm{b}, *}$, Ming-Sheng Shang ${ }^{\mathrm{b}, \mathrm{c}}$, \\ Chi Ho Yeung ${ }^{\mathrm{d}, *}$ \\ a Physics and Photoelectricity School, South China University of Technology, Guangzhou, 510640, China \\ ${ }^{\mathrm{b}}$ Department of physics, University of Fribourg, Fribourg, $\mathrm{CH}-1700$, Switzerland \\ ' Chongqing Institute of Green and Intelligent Technology, Chinese Academy of Sciences, Chongqing, 400714, China \\ ${ }^{\mathrm{d}}$ Department of Science and Environmental Studies, Hong Kong Institute of Education, Hong Kong \\ ${ }^{\mathrm{e}}$ College of Computer Science and Software Engineering, Shenzhen University, Shenzhen 518060, China
}

\section{H I G H L I G H T S}

- We identified the general classification: vested interests and non-vested interests in competition systems.

- We use contrary behaviors: to be minority or majority to model the different characters between the informed and uninformed investors.

- We find the periodic fluctuation competition inter- and intra-groups in the dynamic progress.

\begin{abstract}
In this paper we analyze the contrary behaviors of the informed investors and uniformed investors, and then construct a competition model with two groups of agents, namely agents who intend to stay in minority and those who intend to stay in majority. We find two kinds of competitions, inter- and intra-groups. The model shows periodic fluctuation feature. The average distribution of strategies illustrates a prominent central peak which is relevant to the peak-fat-tail character of price change distribution in stock markets. Furthermore, in the modified model the tolerance time parameter makes the agents diversified. Finally, we compare the strategies distribution with the price change distribution in real stock market, and we conclude that contrary behavior rules and tolerance time parameter are indeed valid in the description of market model.
\end{abstract}

\section{Introduction}

Nature is a huge complex system with many kinds of competitions and collaborations. Financial markets are typical systems with competition, which play a very important role in modern economics. They have attracted many researchers from various fields to study the mechanism including the stylized facts emerging from collective behavior underlying the

\footnotetext{
* Corresponding authors.

E-mail addresses: yuxiafeiwu@163.com (Y.-X. Zhang), hao.liao@unifr.ch (H. Liao), matus.medo@unifr.ch (M. Medo), chyeung@ied.edu.hk (C.H. Yeung).
} 
Table 1

Rules of being winner or loser.

\begin{tabular}{lll}
\hline & Un-agents & In-agents \\
\hline Winner & In the majority side of in-agents & In the minority side of un-agents \\
Loser & In the minority side of in-agents & In the majority side of un-agents \\
\hline
\end{tabular}

markets, such as peak-fat-tail non-normal behavior, long range correlation, and volatility clustering [1-3]. Unlike in many physical systems, we have no direct way to gain insights into the nature of microscopic interactions in financial markets, thus our understanding on their underlying mechanism remains rather limited and ambiguous. As a result, approaches originally developed to understand complex physical systems are adopted to analyze financial markets.

Among the existing approaches, agent-based approach has become one of the key tools, resembling many-body interactions in physics [4-7]. Compared to conventional quantitative analysis, these approaches aim at revealing a qualitative understanding on the mechanism underlying financial market. The minority game is such an example for describing the competition systems [8,9]. While many interesting phenomena are observed in minority game, it failed to provide a correct picture of the financial markets in other perspective. To make the models more like real financial markets, various influence can be considered, such as relation network, information transmission, communication, and learning progress etc. [10-12]. Various types of agents are also modeled, such as fundamentalists, chartists and noisy traders, momentum traders and reverse momentum traders [13-15]. While the models become more realistic, they usually end up being too complex for thorough understanding and analytical treatment. It is thus necessary to build an appropriate simple model to study the inherent character of the financial markets.

Specifically, when an individual becomes a member of a group, her behavior may change to a behavior consistent with the group, and is contrary to her original personal intention [16]. Similarly, in financial markets, groups and individuals may have contrary aims. For example, in European and America stock markets, the investors are classified into informed investors and uninformed investors [15]. The informed investors exploit the insider information to make profits, they buy or sell, and wish other investors to choose the opposite action (i.e. respectively sell or buy). In other words, the informed investors want to take an action where minority of the uninformed investors take. On the contrary, the uninformed investors want to follow the action of the informed investors, and the uninformed investors want to take an action where majority of the informed investors take. Similarly, in the Chinese stock market, investors are classified into bankers and retailers, whose behaviors are similar to those of the informed investors and the uninformed investors. In different systems, the classification of the two opposite group is not the same, such as vested class and non-vested class, ruling class and ruled class, bakers and retailers, etc.

In this paper, we will introduce a model where the agents (such as the informed and uninformed investors) perform according to the former mentioned behaviors. These contrary behaviors of the two groups of agents lead to a hierarchy of inter- and intra-community competition. Interesting phenomenon such as a periodic fluctuation of strategies among agents is observed in the model. The distribution of strategies also shows a prominent central peak relevant to the characteristic distribution of price change in stock markets. The model hence deepens our understanding of competition among investors in stock markets.

\section{Model}

Specifically, we introduce $N$ agents to simulate a competition system. The stock market is a typical financial competition system, and we will construct the model based on the stock market concept. The investors are classified into informed investors and un-informed investors in European market. Then the model consists of $N_{i}$ informed agents (in-agents) and $N_{u}$ uninformed agents (un-agents). We set both $N_{i}$ and $N_{u}$ to be odd numbers to avoid a tie of on deciding the minority or majority side, such that $N_{i}+N_{u}=N$ is an even number. In the market, it is bad thing for the informed investors that if many uninformed investors see through what the informed investor are tend to do(or choose). Correspondingly, in the model, in-agents and un-agents stand for two kinds of agents with different behaviors: in-agents try to hide their action from the un-agents, and we model this behavior by rewarding in-agents if they take the same action as minority of the un-agents take, otherwise they lose. On the contrary, un-agents try to follow the action of the in-agents, and we model this behavior by rewarding un-agents if they take the majority action of the in-agents take, and otherwise they lose $[17,18]$. We denote that the proportions of un-agents and in-agents among the whole population by $\rho_{u}=N_{u} / N$ and $\rho_{i}=N_{i} / N$, respectively.

For all agents to make up their choices, each of them is characterized by a strategy(gene value) $g$, which is a random number in $[0,1]$ [19-21]. In each time step, all agents choose ' 0 '( with a probability $1-g$. After all the agents have made a choice, one can obtain the number of un-agents who choose the ' 0 ' or the ' 1 ' actions, as well as the number of in-agents who choose the ' 0 ' or the ' 1 ' actions. An un-agent is rewarded with one point $r=1$ if she chooses the majority choice of the in-agents, and otherwise loses one point $r=-1$ [22-24]. On the other hand, an in-agent is rewarded with one point $r=1$ if she chooses the minority choice of the un-agents and otherwise she loses one point $r=-1$. A summary is found in Table 1 . At the end of each round, if an agent's score falls below a critical value $d=-4$, its strategy is replaced [19], by a newly drawn strategy in [0,1], and the score is reset to zero.

Although the score counts the number of times an investor wins or loses, it does not directly represent profit. We do not introduce a measurement of profit to our model since it would lead us to define many other rules and variables, such as funds, 
stocks, price etc., and corresponding rules, which will complicate the model. In our model, agents modify their strategies, and different strategies have different lifetimes. If a strategy has not been replaced for a long time steps, the strategy has a long lifetime. In this paper, we will examine the strategy distribution, which is a distribution of strategies held by the agents at a particular time instant. Strategies which show high occurrence in the distribution, e.g. a peak, are strategies with long lifetimes. They can be considered as safe strategies, and conservative agents may like to keep the safe strategies for long time steps. In other words, strategies with long lifetimes can be regarded as low risk or safe strategies, while the strategies with short lifetimes can be considered as risky strategies in some extent. In systems to study competition, profit has a relation with risk: high risk is usually associated with large profit, and low risk is usually associated with small profit $[25,26]$. Based on the relations between strategies, risk and profit, we will only study the distribution of strategies to draw insights on the distribution of risk and profit.

\section{Simulation and analysis}

Since there are un-agents and in-agents, we will show the strategy distribution separately for the two kinds of agents. Since the agents' strategies are replaced when their scores are lower than a threshold, the strategy distribution is continuously updated, and we thus show the strategy distributions as a function of time. Fig. 1 shows an example of strategy distributions of the un-agents (a) and in-agents (b) for 80000 time steps after $2 \times 10^{6}$ time steps. Here, the total number of agents is $N=10000$, the proportion of un-agents $\rho_{u}=0.5999$. As long as the proportion of un-agents is not too small, the strategy distributions are similar to Fig. 1 for a large range of $\rho_{u}$. On the other hand, when the proportion of un-agents is small enough, the group character for un-agents disappears, and the strategy distribution for un-agents as a function of time appears to be a discrete and random distribution (see Appendix B). That is when the number of un-agents is small enough, their actions are short of collective and average behavior, which leads to a large fluctuation in the distribution of the agents. In our model, there are two kinds of competitions: competition among agents and competition between the un-agents and in-agents groups. When the proportion of the un-agents or the in-agents is very small, the group character disappears, so in most cases latter we do not discuss the extreme cases in detail, and we will give some analysis about the extreme case in Appendix.

First, we compare Fig. 1(a) and (b), the strategy distributions among the un-agents and in-agents respectively. Although the behaviors of the two groups of agents are completely opposite, i.e. one group prefers minority while the other prefers majority, yet it is interesting that their strategy distributions are similar, except for a phase shift. The phase of the strategy distribution of the un-agents is ahead of that of the in-agents by a quarter period, where each period is composed of about 8000 time steps for this set of parameters. If Fig. 1(a) translates a quarter of period backward, the distribution becomes almost the same with Fig. 1(b).

Because the two distributions only differ by a phase shift, we will analyze in detail the distribution in Fig. 1(a) only. The most obvious and interesting characters are the periodic fluctuation and the prominent peak at the middle strategy. In traditional Minority Game and its variants, competition exists among individual agents, such that every agents want to be in the minority. While in our model, the competition does not only exist among individuals, but it also exists between the two groups, i.e. between the un-agents and the in-agents groups. We can imagine that in each group there are both winners and losers, and the ratio of the number of winners and losers in each group is fluctuating with time. When the success rate in un-agents group is higher than the failure rate, the un-investor group is in an advantageous position. At the same time, the failure rate in in-agents group must be higher than the success rate, and the in-agents group is in a disadvantageous position, and vice versa. In other words, a large success rate in one group must be associated with a lower success rate in the other group, creating a frustration between the two groups. In the long run, the two groups compete to be the advantageous group in a periodic manner, and the strategy distributions of the two groups differ by a phase shift.

Other than the periodic behavior, we can also see from Fig. 1(a) that there is a peak at the middle strategy $g=0.5$, so we call the middle strategy as peak strategy to emphasize its stability in the distribution. To further understand Fig. 1 , we show in Fig. 2 the change of strategy distributions as a function of time of un-agents at different strategies $g=$ $0.01,0.45,0.48,0.49,0.5$. It can be seen that the strategy distributions are periodic, with a period of roughly $T=32000$ time steps. The proportion of strategies differ greatly from $g=0.5$ oscillates with a larger amplitude, while the proportion of strategies close to $g=0.5$ oscillates with a smaller amplitude. At $g=0.5$, the proportion of agents is high and almost stable, which behaves like a stable peak. In the model, since the probability $g$ to buy and the probability $1-g$ to sell are equal at $g=0.5$, the agents with $g=0.5$ have equal probability to win or lose since the occurrence no matter which side is more likely to be the winning side. On the other hand, the probability $g$ to buy and the probability $1-g$ to sell are different when the strategies are away from $g=0.5$. At certain time, the agents with strategies away from $g=0.5$ are more likely to win, but after a period of time they become more likely to lose. In this case, agents with strategy different from $g=0.5$ change their strategies periodically to keep up with the winning side. The larger the difference from $g=0.5$, the more the difference between the buying and selling probabilities, and the larger the amplitude of oscillation.

Fig. 3 shows the strategy distributions of un-agents at some typical time steps. The strategy distributions at the 0th and the 16000th step are not symmetrical, but are mirror images to one another. It again shows the periodic fluctuation of strategies, as the distribution develops into its mirror image as time evolve for half a period. The peak character at strategy $g=0.5$ in Fig. 3 corresponds to the peak observed in Fig. 1(a), which is relevant to the important universal character of a central prominent peak in the stock price distribution in stock markets. More discussion will be given later when we describe 

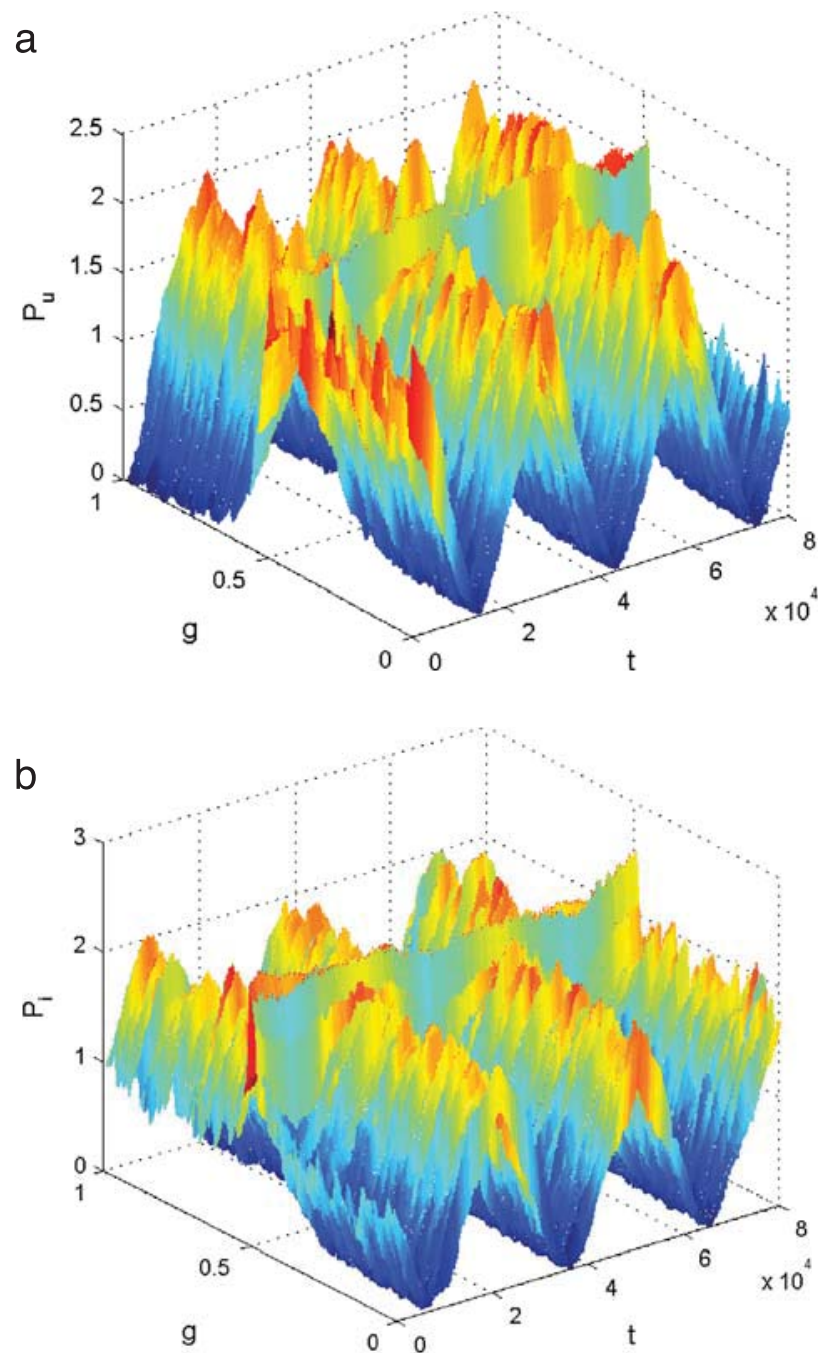

Fig. 1. The strategy distributions of the un-agents (a) and in-agents (b) along the time steps and gene values. $N=10000, \rho_{u}=0.5999$.

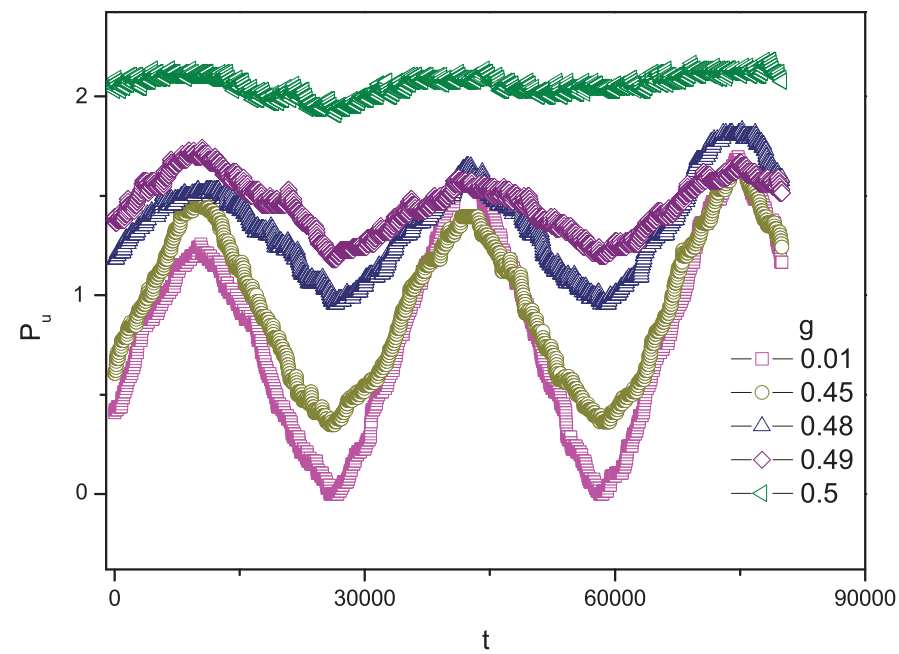

Fig. 2. The strategy distributions of the un-agents along the time steps at some special gene values. $N=10000, \rho_{u}=0.5999$. 


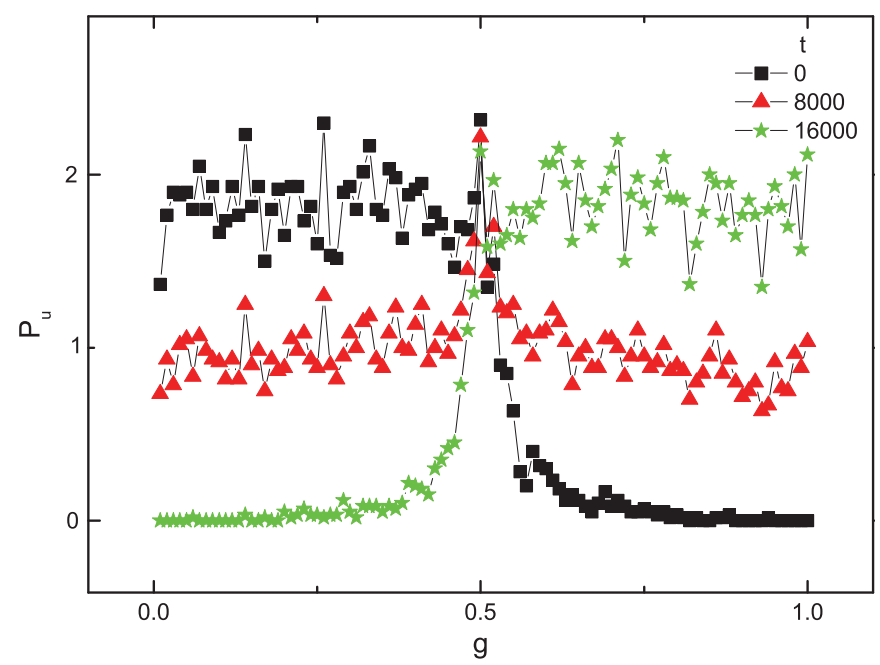

Fig. 3. The strategy distributions of the un-agents along the gene values at some typical time steps. $N=10000, \rho_{u}=0.5999$.

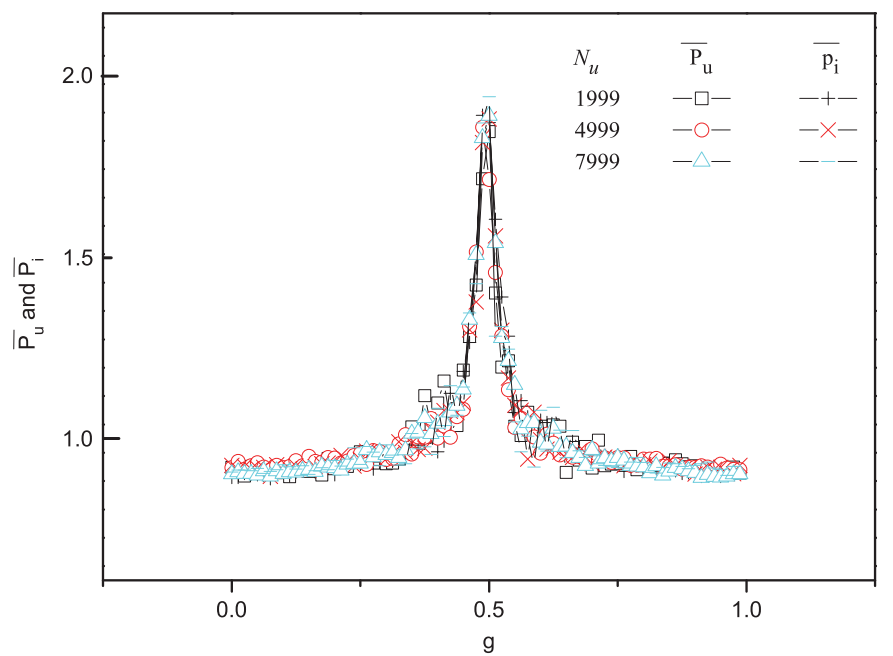

Fig. 4. The average strategy distributions of the un-agents and the in-agents. $N=10000$

Fig. 7. In Fig. 3, the peak in the distribution is almost stable over time, while the two ends of the distribution go up and down in reverse directions like the seesaw.

\section{Simulation and discussion}

Compared to previous results which show the strategy distributions as a function of time, Fig. 4 shows the time-averaged distributions of un-agents $\overline{P_{u}}$ and in-agents $\overline{P_{i}}$ for different ratios of un-agents. Here, the total number of agents is $N=10000$. There are four major observations. First, the agents congregate around the peak strategy $(g=0.5)$. It means that the peak strategy has a longer lifetime than the other strategies. Second, the time-averaged strategy distributions are almost the same for the un-agents and the in-agents. Third, the time-average strategy distributions are almost the same for a large range of proportion of the un-agents $\left(\rho_{u}=0.1999,0.4999,0.7999\right)$ as long as $\rho_{u}$ is not too small. When the proportion of un-agents is very small, their actions are not from a collective behavior which lead to a discrete distribution (see Appendix B). In a word, the strategy distributions are robust against a wide range of parameters, such as the proportions of the un-investor.

What is the key mechanism in the model which leads to the central prominent peak in the strategy distribution? As we know, a prominent peak and fat tails are observed among the price change distribution in many real stock markets, which suggest that this behavior is independent on the initial conditions and the characteristics of the stock markets, and is induced by the inherent market mechanism. In Fig. 4, the strategy distributions with peak non-normal character are independent of the proportions of the two groups of agents, since the two groups only interact and communicate through their collective action, and a large enough group which leads to a reliable collective behavior within the group is already sufficient to lead the overall model dynamics. Compared to the widely studied model of Evolutionary Minority Game (EMG) [19], the strategy 


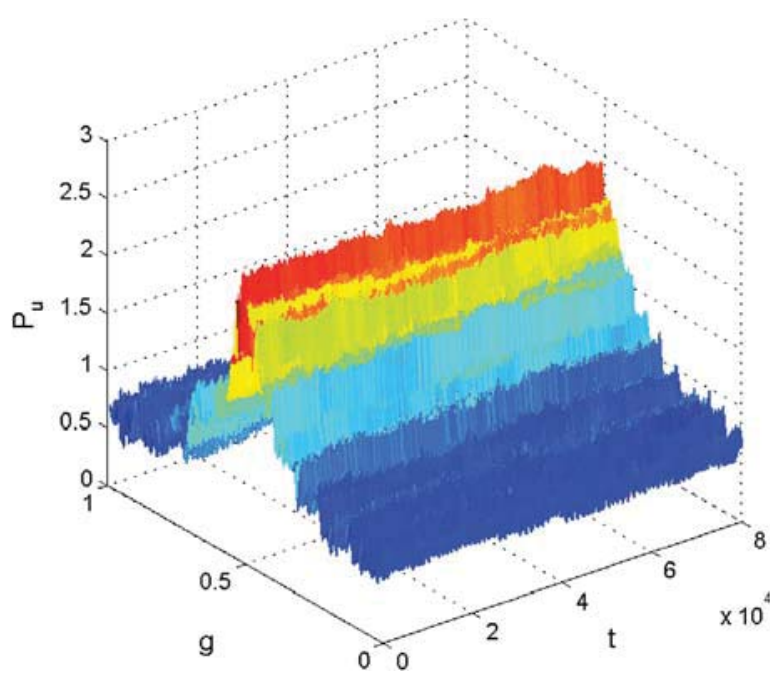

Fig. 5. The strategy distributions of the un-agents along the time steps and gene values. $N=10000, \rho_{u}=0.5999, K=10000$.
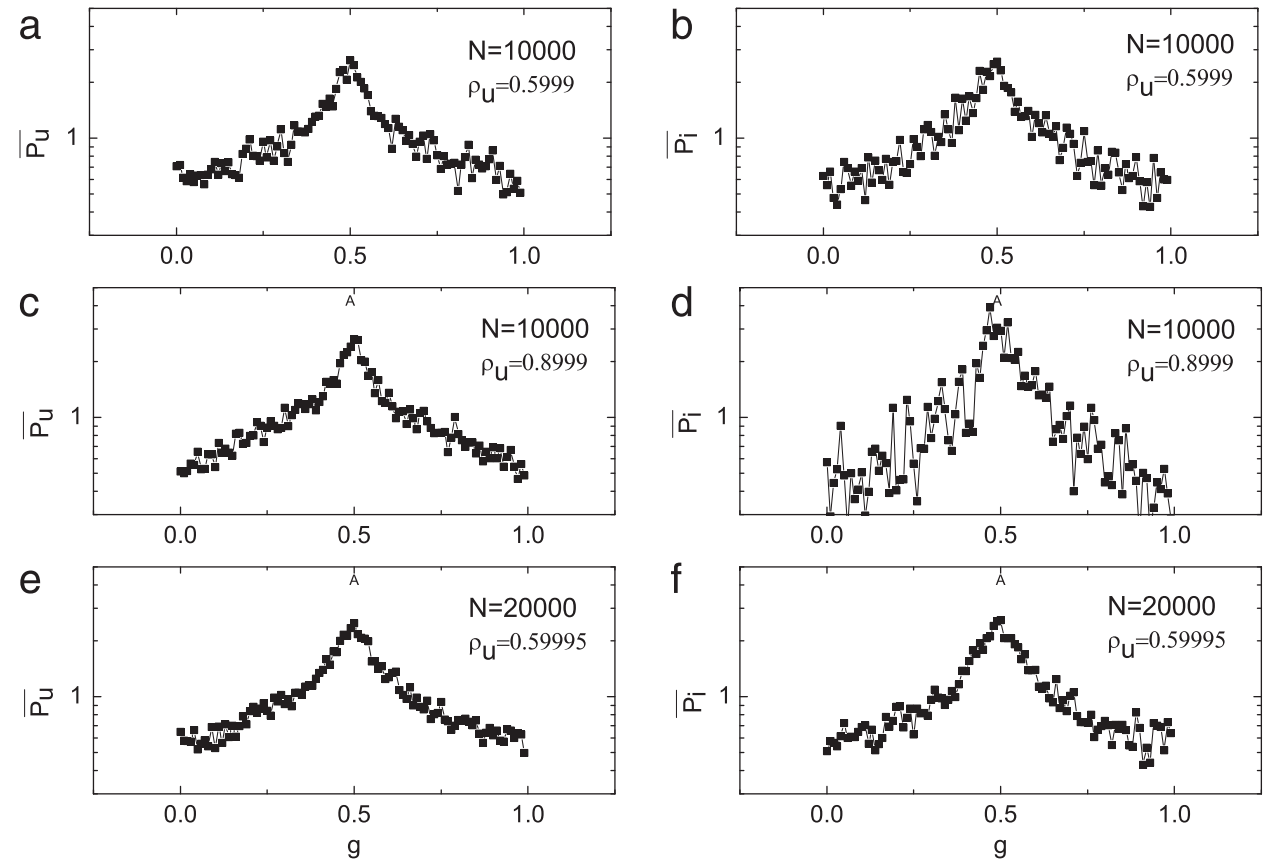

Fig. 6. The average strategy distributions of the un-agents and in-agents for different cases. $K=10000$.

distribution is ' $U$ ' shape with the same parameters, different from the observed peak of the strategy distributions in the present model. The main difference between the two models is that there are two kinds of agents in our model and they compete to be either in the minority or the majority side of the other group, which is different from the simple one-group competition to be in the minority side in EMG. The competition between the two groups in our model is important to produce the peak in the strategy distribution.

\section{Modified model}

As we have seen, the two groups win alternately in a periodic manner, such that the agents in the advantageous group may win for a large number of time steps, and those in the disadvantageous group may lose for a large number of time steps. The winners in one group then become losers in the next period, who then become the winners again in the coming period.

Nevertheless, the mechanism by which the agents change their strategies may seem unrealistic. For example, if an agent is in the advantageous group and wins for a large number of time steps (e.g. 1000 time steps), his score becomes a large number (e.g. 1000). Then, if the advantageous group becomes the disadvantageous group in the coming period, the agent 

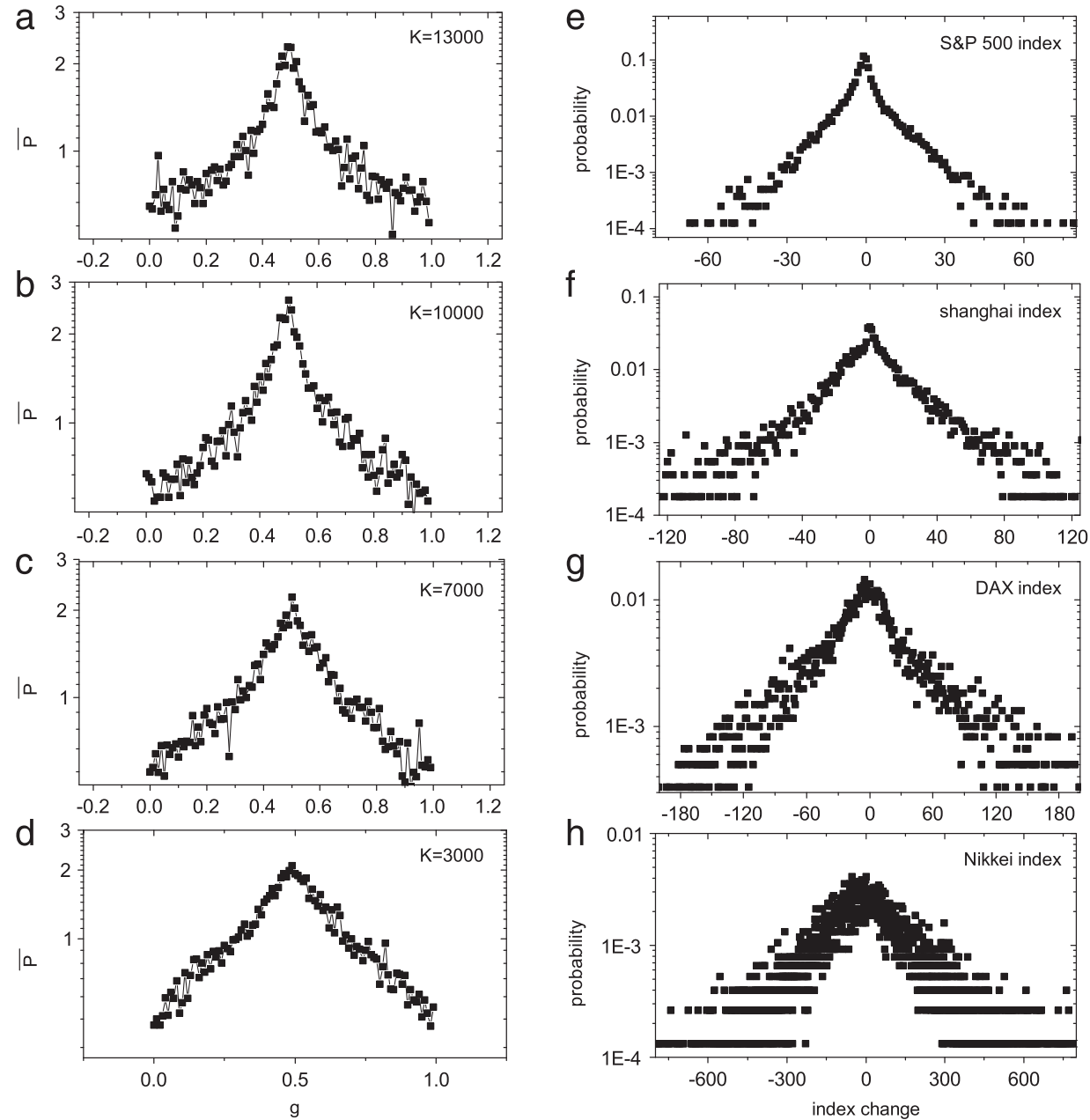

Fig. 7. The semi-log strategy distributions of all agents $\bar{P}$ as a function of gene values for different $K$ (a-d), and four semi-log index difference distributions in real markets $(\mathrm{e}-\mathrm{h})$.

loses for a large number of time steps (e.g. 1005 time steps) until his score decreases gradually below the critical value $d=-4$. In reality, agents would not wait for such a large number of losing time steps until their scores are below the critical value before changing their strategies. Thus, the original mechanism for changing the strategies may seem unrealistic. As a result, we add a new mechanism for the agents to change their strategies: if an agent $i$ loses continually for $k_{i}$ time steps, the agent changes his strategy. Taking into account the heterogeneity of agents, different agents have different tolerance times $k_{i}$. For simplicity, the tolerance times are drawn from a uniform distribution $[1, K]$, where $K$ is a parameter to produce the investor's tolerance time $k_{i}$.

\section{Simulation and analysis}

By implementing the new rule in modifying the strategy, Fig. 5 shows the strategy distribution of the un-agents for 80000 time steps after $2 \times 10^{6}$ time steps at $K=10000$. Since the strategy distribution of the in-agents is similar to that of the unagents, and we only show the strategy distribution of the un-agents as an example. Compared with periodic fluctuation in Fig. 1, such periodic dynamics does not exist in Fig. 5. In addition, the peak in the strategy is also obvious in Fig. 5. The reason is that by implementing the new rule, the agent has more freedom to change his strategy at any time during the simulation, and the periodic dynamics disappears seemingly when the number of agents in each group is large enough. Actually, the periodic character does not disappear in nature (see Appendix). In the modified model, agents can switch their strategies more frequently which favor the peak of strategies at $g=0.5$. Fig. 6 shows the time-averaged strategy distributions for different cases. It can be seen that with the same parameter $K$, the average strategy distributions are similar for un-agents (left column) and in-agents (right column), and the different number and proportion of un-agents and in-agents.

In our model, the total number of agents $N$ may correspond to the scale of the market. We observe that when $N$ is large enough, the stable strategy distribution is achieved from different system size $N$, suggesting that the market behaviors 

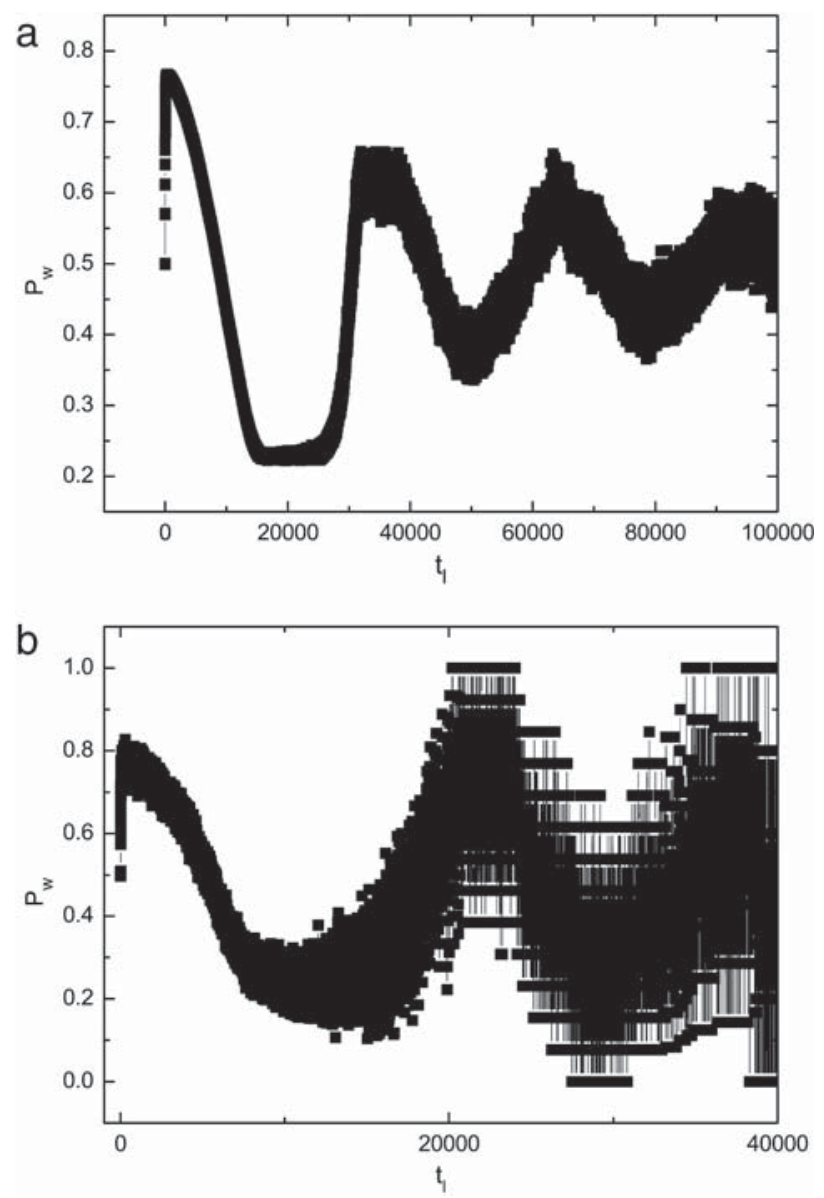

Fig. 8. The win probability of the agents in original model (a) and modified model (b) at different lifetimes. $N=10000, \rho_{u}=0.5999$.

are independent of large market scale. This is consistent with empirical observations. On one hand, there are many selfgoverned stock markets with different scale, yet the price difference distributions in different stock markets have similar characters. Moreover, as long as the proportion of un-agents is not extremely small, the strategy distributions converge to the universal distribution which is consistent with the observations in real markets. On the other hand, self-governed stock markets have different characteristics, such as the proportion of various agents, un-investor and in-investor, and yet the emergent macroscopic behaviors are always similar.

Fig. 7(a)-(d) show the strategy distributions of all agents $\bar{P}$ in a semi-log scale at different values of $K$. The shapes of these distributions are compared to those in Fig. 7(e)-(h), which show several examples of the distribution of index changes in semi-log scale obtained from real markets. These distributions of index changes are computed at a one-day interval using the daily closing prices in S\&P 500 index (from 1975.1 to 2014.9), Shanghai index (from 1990.12 to 2014.9), DAX index (from 1990.11 to 2014.9), and Nikkei index (from 1984.1 to 2014.9). Although the two distributions are different in nature, their shapes are similar and are indeed relevant to one another. In Fig. $7(\mathrm{a})-(\mathrm{d})$, the strategies around $g=0.5$ are conservative strategies, which are less risky but may lead to less profit. On the other hand, the strategies at the two ends of the distribution are more risky strategies, and may lead to larger profit or loss. While in Fig. 7(e)-(h), small index changes may correspond to the occurrence of small returns, while larger index changes correspond to larger profit or loss. As a result, the two seemingly different distributions are related to one another. This further suggests that our simple model can indeed capture the essence of competition between informed and uninformed agents in stock markets.

\section{Conclusion}

In summary, we introduced a model to reveal the competition between informed and uninformed agents in stock markets. These two groups of agents compete within and between the groups in the model, leading to an interesting dynamics of alternating winning and losing period for both groups. By measuring the distribution of the strategies among the agents, we found that the less risky strategies are much more popular than the more risky but more profitable strategies. Such behaviors are robust against the system size as well as the proportion of informed and uninformed agents. Finally, we 

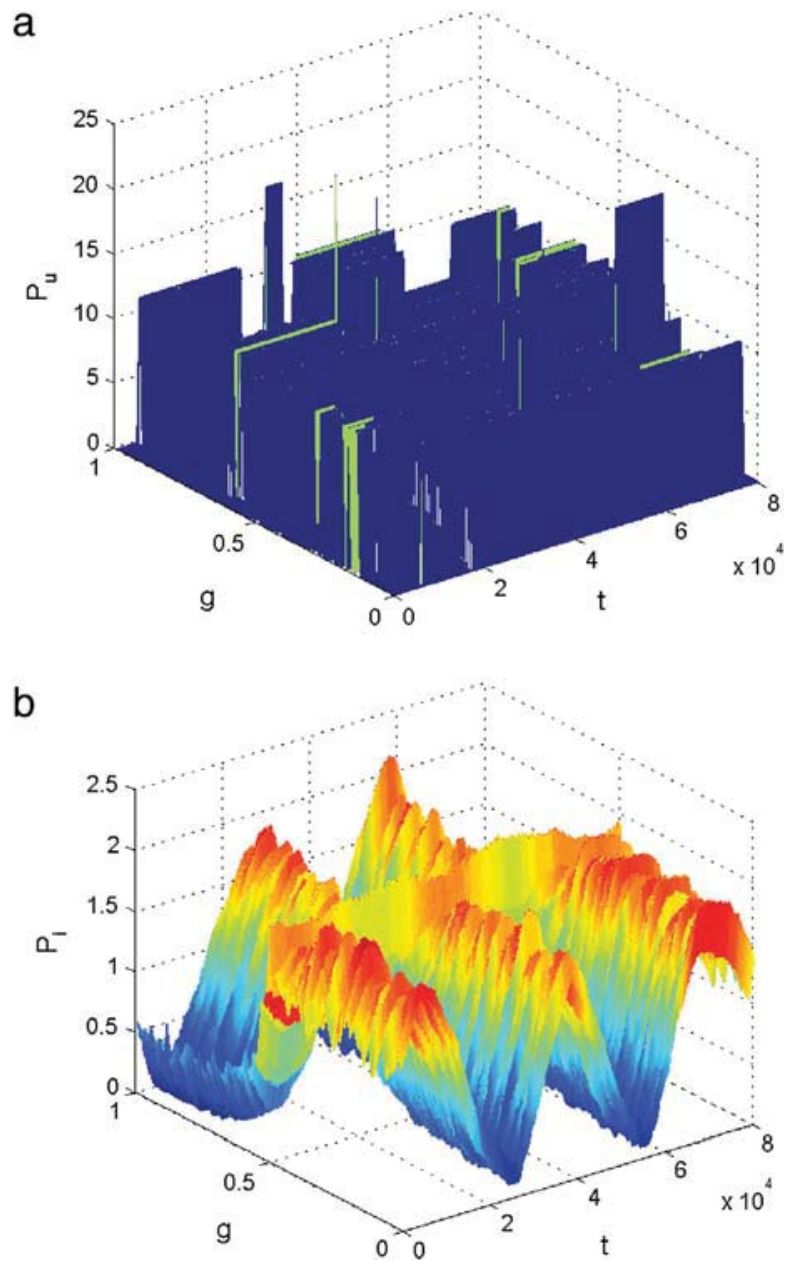

Fig. 9. The strategy distributions of the un-agents (a) and in-agents (b) along the time steps and gene values in the original model. $N=10000, \rho_{u}=0.0009$, $d=-4$.

draw relevance between our results and the price change distribution in real stock markets, and draw insights to explain the prominent central peak in the empirical distributions. Our results suggest that the introduced model captures and reveals the essence of complex competition in stock markets.

\section{Acknowledgments}

We thank Prof. Yi-Cheng Zhang for fruitful discussion and comments. This work was supported by the Fundamental Research Funds for the Central Universities (Grant Nos. 2014ZM0079, $2014 Z Z 0070$ and 2015ZZ056), National Natural Science Foundation of China (Grant Nos. 61503140, 11205061, 11247253, 11504114, 11547040, 61176061 and 61474046), the EU FP7 Grant 611272 (project GROWTHCOM) and the China Scholarship Council. CHY is partially supported by the Internal Research Grant RG71/2013-2014R of HKIEd, Science and Technolgy Innovation Commission of Shenzhen (No. JCYJ20150625101524056).

\section{Appendix A. How can the new strategies compete with old ones?}

In the model, scores are cumulative quantities, but when a strategy is replaced with a new one, the agent's score is reset to zero. How can the new strategies compete with old ones? To illustrate this question, we calculate the win probability $P_{W}$ of the strategies at different lifetimes $t_{l}$, where small lifetime means that the strategy is a new strategy. Fig. 8(a) and (b) show the win probability of strategies at different lifetimes for the original model and the modified model, respectively. In Fig. 8, the win probability for new strategy is about 0.5 , and it increases very soon and then decreases etc. There is a periodic character for win probability $P_{W}$. The new strategy is a random number is the range $[0,1]$ which is symmetry, so the win probability for new strategy is 0.5 . The score of a new strategy is zero, the score of a bad strategy will soon decrease 
a
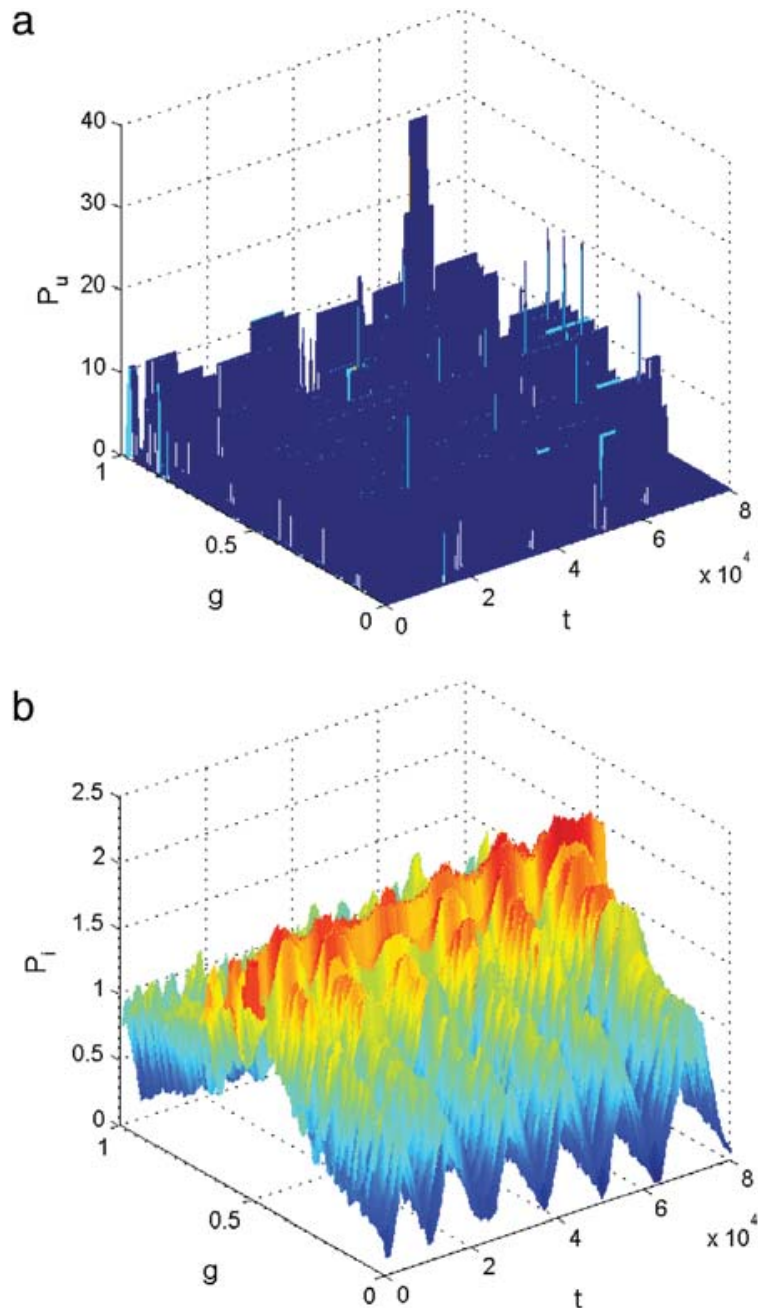

Fig. 10. The strategy distributions of the un-agents (a) and in-agents (b) along the time steps and gene values in the modified model. $N=10000$, $\rho_{u}=0.0009, d=-4, K=10000$.

to the critical value $d$, i.e. the bad strategy will soon be replaced and the good new strategy survives. After a while the win probability will increase. But the competition is dynamic, the good situation for a strategy at this time will change to a bad situation later, then after some time steps the win probability will decrease, and so forth. The difference between Fig. 8(a) and (b) is that there are many periods in Fig. 8(a) and limited period in Fig. 8(b). The reason is that the lifetime of a strategy in modified model is more limited than that in the original model. For Fig. 8(b), the large lifetime is about 4000 time steps. So when the lifetime is large enough, there are very few agents, and the average error is very large which can be seen from Fig. 8(b).

\section{Appendix B. The extreme case: the strategy distribution when $\rho_{u}$ is very small}

For better understanding of the extreme case of our model, Figs. 9 and 10 illustrate the strategy distribution for the original model and the modified model at $\rho_{u}=0.0009$. The note is that in the extreme case the strategy distributions are different for un-agents and in-agents. Therefore, the strategy distributions for un-agents (Figs. 9(a) and 10(a)) and in-agents (Figs. 9(b) and 10(b)) are shown separately. The reason for the difference is that the average error is very large for the group with very small number of agents. In the extreme case, the strategy distribution for un-agents is random (see Figs. 9(a) and 10(a)). Because the un-agents compete with the in-agents, the average error will infect the strategy distribution of in-agents too. For example, in the modified model, the strategy distribution of the in-agents show period character (see Fig. 10(b)) which is different from that at middle $\rho_{u}$ (shown in Fig. 5). The reason is that the period character of the un-agents is short of average when $\rho_{u}$ is small, and results in the reappearance of the periodic strategy distribution of the in-agents.

Besides the periodic character in Figs. 1-3, we can see from Fig. 8 that the win probability with different lifetimes shows periodic character, that is to say the judgement of a strategy changes with time, which depends on the whole situation. Based 
on Figs. 9 and 10, in the extreme case, the strategy distributions of the in-agents show periodic character too. Especially, Fig. 10(b) shows periodic character in the modified model too. In a word, the periodic character is important in our model including the modified model. The period character in this paper is the dynamic process of the model, it can be seen from Figs. 1, 2, 3, 8, 9 and 10. Based on these figures, we can imagine the periodic dynamic process. The model is composed of two groups of agents with contrary behaviors. The contrary behaviors will drive the model fluctuate periodically, i.e. no group will always be in the advantage. That is the competition.

\section{References}

[1] T.A. Schmitt, R. Schäfer, M.C. Münnix, T. Guhr, Europhys. Lett. 100 (2012) 38005.

[2] S. Mike, J.D. Farmer, J. Econom. Dynam. Control 32 (2008) 200-234.

[3] V. Gontis, A. Kononovicius, PLoS One 9 (2014) e102201.

[4] T. Lux, M. Marchesi, Nature 397 (1999) 498.

[5] J.D. Farmer, D. Foley, Nature 460 (2009) 685

[6] A. Chakrabortia, I.M. Tokea, M. Patriarcabc, F. Abergela, Quant. Finance 11 (2011) 1013.

[7] A. Kononovicius, V. Gontis, Europhys. Lett. 101 (2013) 28001.

[8] D. Challet, Y.C. Zhang, Physica A 256 (1998) 514.

[9] D. Challet, M. Marsili, Phys. Rev. E 60 (1999) R6271

[10] R. Lye, J.P.L. Tan, S.A. Cheong, Physica A 391 (2012) 5521.

[11] T. Preis, S. Golke, W. Paul, J.J. Schneider, Europhys. Lett. 75 (2006) 510.

12] B. LeBaron, J. Econ. Behav. Organ. 83 (2012) 424.

[13] M. Gilli, P. Winker, Comput. Statist. Data Anal. 42 (2003) 299.

[14] M. LiCalzi, P. Pellizzari, Quant. Finance 3 (2003) 470.

15] R. Bloomfield, W.B. Tayler, F.H. Zhou, J. Finance 64 (2009) 2535.

[16] L.B. Gustave, The Crowd: A study of the popular mind 1895.

17] F. Lillo, E. Moro, G. Vaglica, R.N. Mantegna, New J. Phys. 10 (2008) 043019.

18] S. Hod, E. Nakar, Phys. Rev. Lett. 88 (2002) 238702.

19] N.F. Johnson, P.M. Hui, R. Johnson, T.S. Lo, Phys. Rev. Lett. 82 (1999) 3360

20] S. Hwang, M. Salmonb, J. Empir. Finance 11 (2004) 585.

21] D. Bernhardta, M. Campellob, E. Kutsoatic, J. Financ. Econ. 80 (2006) 657.

[22] K. Yuan, J. Finance 60 (2005) 379.

$23]$ R.A. Lambert, C. Leuz, R.E. Verrecchia, Rev. Finance 16 (2012) 1.

24] S. Alfarano, T. Lux, F. Wagner, Comput. Econ. 26 (2005) 19.

25] H. Guo, R.F. Whitelaw, J. Finance 3 (2006) 1433.

[26] J.T. Scruggs, G. Paskalis, J. Finan. Quant. Anal. 38 (2003) 295. 